\title{
Correction to: Exercise following breast cancer: exploratory survival analyses of two randomised, controlled trials
}

\author{
S. C. Hayes ${ }^{1} \cdot$ M. L. Steele ${ }^{1} \cdot$ R. R. Spence ${ }^{1} \cdot$ L. Gordon ${ }^{2} \cdot$ D. Battistutta ${ }^{1}$.

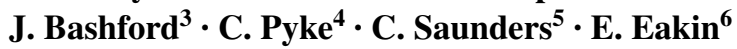

Published online: 10 November 2017

(C) Springer Science+Business Media, LLC, part of Springer Nature 2017

Correction to: Breast Cancer Res Treat https://doi.org/10.1007/s10549-017-4541-9

In the original publication of the article, under the heading Discussion, 1st paragraph, the sentence that reads as, "Nonetheless, our observed improvements of over $50 \%$ for OS and over $30 \%$ for DFS (HRs: 0.45 and 0.66 , respectively) are consistent with results from other available studies" should read as "Nonetheless, our observed improvements of over
50\% for OS and DFS (HRs: 0.45 and 0.66 , respectively) are consistent with results from other available studies."

Under the heading Discussion, 3rd paragraph, the sentence that reads as "We cannot discount the possibility ... such as education, income and access to care [1,7]" should read as "We cannot discount the possibility... such as education, income and access to care, which ultimately have on survival outcomes $[1,7]$."

The original article can be found online at https://doi.org/10.1007/s10549-017-4541-9.

S. C. Hayes

sc.hayes@qut.edu.au

1 Institute of Health and Biomedical Innovation, School of Public Health and Social Work, Queensland University of Technology, Brisbane, Australia

2 QIMR Berghofer Medical Research Institute, Brisbane, Australia

3 Wesley Hospital, Brisbane, Australia

4 Mater Public and Private Hospital, Brisbane, Australia

5 University of Western Australia, Perth, Australia

6 School of Public Health, Cancer Prevention Research Centre, The University of Queensland, Brisbane, Australia 